\title{
Peroxydation lipidique et vieillissement cérébral : l'apport des études épidémiologiques
}

\author{
Pascale BARBERGER-GATEAU ${ }^{1}$ \\ Cécile DELCOURT ${ }^{\top}$ \\ Claudine BERR ${ }^{2}$ \\ ${ }^{1}$ Inserm U593 Épidémiologie, \\ Santé publique et développement ; \\ Université Victor Segalen Bordeaux 2 ; \\ 33076 Bordeaux cedex, France \\ $<$ Pascale.Barberger-Gateau@isped.u- \\ bordeaux2.fr> \\ ${ }^{2}$ Inserm E0361 Pathologies du système \\ nerveux : recherche épidémiologique et clinique ; \\ Université Montpellier I ; Hôpital La Colombière ; \\ 34093 Montpellier Cedex 5, France. \\ Fax : 0557571486.
}

Le vieillissement cérébral pathologique génère un fardeau économique et social considérable. L'étude PAQUID (QUID des personnes âgées) estime que $17 \%$ des personnes âgées de 75 ans et plus sont atteintes de démence [1], ce qui représenterait environ 870000 cas actuellement en France avec une incidence de 220000 nouveaux cas par an (source : rapport à I'Office parlementaire pour l'évaluation des politiques de santé, juin 2005). La cause la plus fréquente de démence est la maladie d'Alzheimer (MA), affection irréversible dans l'état actuel de nos connaissances puisque les seuls traitements disponibles sont purement symptomatiques [2]. Les principaux facteurs de risque de MA, l'âge et la possession de l'allèle $\epsilon 4$ du gène de l'apolipoprotéine $E$ (apoE4) pour sa forme tardive ou sporadique, sont des facteurs de prédisposition qui n'offrent aucune possibilité de prévention. II importe donc d'identifier des facteurs environnementaux modulant le vieillissement cérébral et le risque de MA, sur lesquels il serait possible d'agir. Avant la survenue du stade de démence irréversible, le déclin cognitif progressif va se manifester par des déficiences cognitives légères (mild cognitive impairment ou $\mathrm{MCl}$ ) qui atteignent environ 15 à $20 \%$ de la population âgée de 65 ans et plus, mais qui représentent un état instable encore potentiellement réversible dans son évolution naturelle ou grâce à la mise en place d'une prévention secondaire [3]. Une stratégie de prévention devrait viser à éviter ou retarder la survenue du passage vers la démence et à favoriser le retour à une cognition normale chez des sujets au stade $\mathrm{MCl}$.

\begin{abstract}
The brain is particularly vulnerable to oxidative stress. Epidemiological studies have provided nutritional and biological arguments for a role of anti-oxidants such as vitamins E and C, flavonoids, and carotenoids against brain aging. There are however discrepancies between studies regarding potentially effective doses, interactions with genetic characteristics or smoking, or efficacy of supplements. A single intervention trial showed a small effect of vitamin E in Alzheimer patients, whereas all primary or secondary prevention trials were negative. Part of these discrepancies may be explained by the absence of simultaneous consideration of the lipid status, whereas most epidemiologic studies have shown a protective effect of long-chain $\omega 3$ fatty acids against cognitive decline or dementia. However, there is no published clinical trial regarding their effect on this outcome. Additional studies are required to identify mechanisms of action and potentially effective doses before giving recommendations for a nutritional prevention of pathological brain aging.
\end{abstract}

Key words: oxidative stress, aging, humans, dementia, Alzheimer disease, omega-3, epidemiology

Cible potentielle d'actions de prévention, le rôle de la nutrition dans le vieillissement cérébral et la MA suscite un intérêt croissant [4], avec des hypothèses physiopathologiques portant plus particulièrement sur l'effet conjoint des anti-oxydants [5] et des acides gras [6]. Nous aborderons successivement ces deux pistes en montrant leur complémentarité, à travers les données d'études épidémiologiques.

\section{Les études épidémiologiques françaises sur nutrition et vieillissement cérébral}

Les études épidémiologiques peuvent apporter des arguments sur les relations entre apports ou statut nutritionnel et vieillissement cérébral. Cependant, seules les études longitudinales (études de cohorte) sont pertinentes pour montrer que le comportement alimentaire observé a bien précédé le déclin cognitif ou la démence, et non l'inverse. La plupart de ces études sont américaines et reflètent donc des habitudes alimentaires particulières tout en comprenant des proportions importantes d'utilisateurs de suppléments, qui peuvent biaiser les résultats si leur composition précise n'est pas prise en compte. Cependant, trois grandes études épidémiologiques françaises comportent à la fois des données nutritionnelles et cognitives répétées en population générale âgée, permettant d'étudier les relations entre nutrition et vieillissement cérébral: l'étude PAQUID [7], l'étude EVA (étude du vieillissement artériel) [8] et l'étude des 3 Cités (3C) [9]. L'étude PAQUID, qui avait pour objectif l'étude du vieillissement cérébral et fonctionnel, a inclus 3777 personnes âgées de 65 ans et plus, vivant à leur domicile, tirées au sort en 1988 dans 75 communes de Dordogne et de Gironde. Les participants ont été visités à leur domicile par une psychologue qui recueillait un ensemble de données sur leur mode de vie, leurs symptômes et leur faisait passer des tests neuropsychologiques. Tous les sujets suspects de démence à l'issue de cette entrevue étaient ultérieurement revus par un neurologue pour confirmer le diagnostic et en préciser l'étiologie. Les sujets ont ensuite été revus à leur domicile environ tous les deux ans selon les mêmes modalités. En 1991-92, nous avons ajouté un bref recueil des habitudes alimentaires, complété par une enquête diététique détaillée (PAQUINUT) sur un sous-échantillon de 169 participants [10]. Des dosages des vitamines $A$ et $E$ plasmatiques, et du malonodialdéhyde (MDA), marqueur de la peroxydation lipidique, ont été effectués dans le plasma de cas de démence incidents et de témoins appariés.

L'étude EVA a inclus 1389 volontaires de Nantes, âgés initialement de 60 à 70 ans en 1991 1993, avec pour objectif principal l'étude du vieillissement artériel. Ils ont été suivis pendant 9 ans avec des tests neuropsychologiques répétés. Différents paramètres de protection antioxydants ont été mesurés initialement dans le plasma (sélénium qui est un cofacteur de la GSH-Px, caroténoïdes totaux) ou les globules rouges (vitamine E). Les thiobarbituric acid reactive substances (TBARS), indicateur de peroxydation lipidique, ont été mesurés dans les globules rouges. Des spectres d'acides gras des 
phospholipides des membranes de globules rouges ont été réalisés 4 ans après l'inclusion sur un sous-groupe de 246 sujets qui ont eu deux évaluations cognitives par le mini-mental state examination (MMSE), un test global des fonctions cognitives.

L'étude $3 \mathrm{C}$ a pour objectif l'étude des facteurs vasculaires de la démence, par le suivi de 9294 personnes âgées de 65 ans et plus vivant à Bordeaux, Dijon et Montpellier, incluses en 1999-2000. Les données nutritionnelles recueillies sur l'ensemble de la cohorte incluaient un bref questionnaire de fréquence de consommation alimentaire, des données anthropométriques et un dosage des lipides plasmatiques (cholestérol et triglycérides). Une biothèque et une DNA-thèque ont été constituées. À Bordeaux, une enquête diététique détaillée a été réalisée auprès de 1800 participants lors du premier suivi. Des dosages des vitamines $A$ et $E$, du MDA et des acides gras plasmatiques sont en cours à partir de la biothèque.

\section{Anti-oxydants et vieillissement cérébral}

Le cerveau est particulièrement vulnérable au stress oxydant en raison de sa composition en acides gras poly-insaturés (AGPI) à longue chaîne très sensibles à la peroxydation (en par- ticulier le DHA C22 : 6(n-3)), de sa consommation élevée d'oxygène entraînant un niveau élevé de production de radicaux libres et de sa teneur en fer-ascorbate pro-oxydant [5]. Par ailleurs, les systèmes de défense anti-oxydants y sont assez peu présents et semblent devenir moins efficaces avec le vieillissement [11]. Dans la MA la protéine $\beta$-amyloïde peut conduire directement à une augmentation de la production de radicaux libres et une augmentation de la peroxydation lipidique y a été observée au niveau cérébral $[5,12]$. Des phénomènes oxydatifs révélés par des dosages d'isoprostanes, marqueurs de la peroxydation lipidique, ont aussi été observés dans les cerveaux de patients atteints de $\mathrm{MCl}$ avec des niveaux similaires à ceux de patients Alzheimer, suggérant un rôle précoce du stress oxydant dans la pathogénie de la MA [13].

Les études épidémiologiques ayant analysé la relation entre consommation d'anti-oxydants et moindre risque de démence ou de MA ont montré des résultats discordants (tableau 1). L'étude PAQUID (flavonoïdes) [7], la Rotterdam Study (vitamines E et C) [14], et la Chicago Health and Aging Study (CHAP) (vitamine E) [15] ont apporté des arguments en faveur d'un rôle protecteur des anti-oxydants nutritionnels contre la démence, contrairement au Washington Heights Inwood Columbia Aging Project (WHICAP) [16]. L'utilisation de supplé- ments en vitamines $C$ ou $E$ était aussi associée à une réduction du risque de démence ou de déclin cognitif dans un sous-échantillon des cohortes Established Populations for the Epidemiologic Study of the Elderly (EPESE) [17], dans la Cache County Study [18] et la Nurses' Health Study [19] mais pas dans le WHICAP [16], ni I'étude CHAP [15], ni dans la Honolulu-Asia Aging Study (HAAS) [20]. De plus, ces relations différaient en fonction du type et/ou du dosage des suppléments, en particulier de l'association entre vitamines $E$ et $C$, mais également après prise en compte d'interactions avec des facteurs environnementaux comme le tabagisme ou génétiques comme l'apoE4.

Les données biologiques confortent les résultats observés avec les données diététiques. Dans l'étude EVA, des taux élevés de TBARS étaient associés à une augmentation de la probabilité de déclin cognitif, d'autant plus que les taux d'antioxydants plasmatiques étaient bas (sélénium, caroténoḯdes ou vitamine E) [8]. Dans PAQUID, des taux bas de vitamine E plasmatique étaient associés à un risque plus élevé de démence ultérieure [21]. Le niveau en anti-oxydants du plasma (activités enzymatiques, vitamines, caroténoïdes) était également plus faible chez des sujets atteints de $\mathrm{MCl}$, et proche de celui des patients Alzheimer [22]. Le rôle spécifique des caroténoïdes xanthophylles dans la MA, la démence vasculaire ou le $\mathrm{MCl}$ est

Tableau 1. Principales études épidémiologiques longitudinales de la relation entre consommation d'anti-oxydants et vieillissement cérébral pathologique.

\begin{tabular}{|c|c|c|c|c|c|}
\hline Étude & $\mathbf{N}$ & Âge & Facteur étudié & Événement suivi & Résultat (après ajustement) \\
\hline $\begin{array}{l}\text { PAQUID } \\
\text { (France) }\end{array}$ & 1367 & $\geq 68$ & $\begin{array}{l}\text { Flavonoïdes } \\
\text { nutritionnels }\end{array}$ & Démence & Effet protecteur \\
\hline $\begin{array}{l}\text { Rotterdam Study } \\
\text { (Pays-Bas) }\end{array}$ & 5395 & $\geq 55$ & $\begin{array}{l}\text { Vitamine E } \\
\text { Vitamine C } \\
\text { Bêtacarotène } \\
\text { Flavonoïdes } \\
\text { Nutritionnels }\end{array}$ & Maladie d'Alzheimer & $\begin{array}{l}\text { Effet protecteur des vitamines } C \text { et } E \\
\text { Pas d'effet du bêtacarotène ni des } \\
\text { flavonoïdes sauf chez fumeurs }\end{array}$ \\
\hline $\begin{array}{l}\text { CHAP } \\
\text { (États-Unis) }\end{array}$ & 815 & $\geq 65$ & $\begin{array}{c}\text { Vitamine } \mathrm{E} \\
\text { Vitamine } \mathrm{C} \\
\text { Bêtacarotène } \\
\text { Nutritionnels + suppléments }\end{array}$ & Maladie d'Alzheimer & $\begin{array}{l}\text { Pas d'effet de la vitamine } C \text { ni du } \\
\text { bêtacarotène, ni de la vitamine } E \text { en } \\
\text { suppléments } \\
\text { Effet protecteur de la vitamine } E \text { alimentaire } \\
\text { seulement chez apoE4 négatifs }\end{array}$ \\
\hline $\begin{array}{l}\text { WHICAP } \\
\text { (États-Unis) }\end{array}$ & 980 & $\geq 65$ & $\begin{array}{c}\text { Caroténoïdes } \\
\text { Vitamine } \mathrm{E} \\
\text { Vitamine C } \\
\text { nutritionnelles + suppléments }\end{array}$ & Maladie d'Alzheimer & $\begin{array}{l}\text { Aucune association avec les données } \\
\text { diététiques ni les suppléments }\end{array}$ \\
\hline $\begin{array}{l}\text { EPESE } \\
\text { (États-Unis) }\end{array}$ & 633 & $\geq 65$ & Suppléments en vitamines $\mathrm{E}$ ou $\mathrm{C}$ & Maladie d'Alzheimer & $\begin{array}{l}\text { Effet protecteur de la vitamine C, pas la E. } \\
\text { Mais peu de variables d'ajustement. }\end{array}$ \\
\hline $\begin{array}{l}\text { Cache County Study } \\
\text { (États-Unis) }\end{array}$ & 3227 & $\geq 65$ & $\begin{array}{l}\text { Suppléments en vitamines } \mathrm{E} \\
\text { (> }>400 \mathrm{UI}) \text { ou C (>500 mg) }\end{array}$ & Maladie d'Alzheimer & $\begin{array}{l}\text { Pas d'association avec l'utilisation de vit. } E \\
\text { ou } C \text { seules mais effet protecteur de } \\
\text { I'utilisation combinée vit. } E+C\end{array}$ \\
\hline $\begin{array}{l}\text { Nurses' Health Study } \\
\text { (États-Unis) }\end{array}$ & 14968 & $70-79$ & $\begin{array}{l}\text { Suppléments en vitamines } \mathrm{E} \text { ou } \mathrm{C} \text {, } \\
\text { sauf multi-vitamines }\end{array}$ & Tests des fonctions cognitives & $\begin{array}{l}\text { Meilleures performances avec suppléments } \\
\text { vit. } E+C \text { combinées, surtout si faible apport } \\
\text { nutritionnel en vit. } E\end{array}$ \\
\hline $\begin{array}{l}\text { HAAS } \\
\text { (hommes japonais à } \\
\text { Honolulu) }\end{array}$ & 2369 & $71-92$ & Suppléments en vitamines $\mathrm{E}$ ou $\mathrm{C}$ & $\begin{array}{l}\text { Démence } \\
\text { Maladie d'Alzheimer } \\
\text { Démence vasculaire }\end{array}$ & $\begin{array}{l}\text { Aucune association avec consommation de } \\
\text { suppléments, à court ou long terme }\end{array}$ \\
\hline
\end{tabular}


Tableau 2. Principales études épidémiologiques longitudinales de la relation entre consommation de lipides et vieillissement cérébral pathologique.

\begin{tabular}{|c|c|c|c|c|c|}
\hline Étude & $\mathbf{N}$ & Âge & Facteur étudié & Événement suivi & Résultat (après ajustement) \\
\hline $\begin{array}{l}\text { Rotterdam Study } \\
\text { (Pays-Bas) }\end{array}$ & 5434 & $\geq 55$ & $\begin{array}{c}\text { Poisson } \\
\text { Lipides et acides gras }\end{array}$ & $\begin{array}{c}\text { Démence } \\
\text { Maladie d'Alzheimer (MA) } \\
\text { Démence vasculaire }\end{array}$ & $\begin{array}{l}\text { Effet protecteur de la consommation de poisson contre } \\
\text { la démence et la MA } \\
\text { Pas d'association avec lipides ni types d'acides gras } \\
\text { consommés }\end{array}$ \\
\hline PAQUID (France) & 1416 & $\geq 68$ & Poisson & $\begin{array}{c}\text { Démence } \\
\text { Maladie d'Alzheimer (MA) }\end{array}$ & $\begin{array}{l}\text { Effet protecteur de la consommation de poisson contre } \\
\text { la démence, à la limite de la signification pour la MA }\end{array}$ \\
\hline CHAP (États-Unis) & 815 & $\geq 65$ & $\begin{array}{c}\text { Poisson } \\
\text { Acides gras } \omega 3\end{array}$ & Maladie d'Alzheimer (MA) & $\begin{array}{l}\text { Effet protecteur de la consommation de poisson, } \\
\text { d'AGPI } \omega 3 \text { totaux et de DHA contre la MA }\end{array}$ \\
\hline CHAP (États-Unis) & 2560 & $\geq 65$ & Lipides & Déclin cognitif (tests) & $\begin{array}{l}\text { Effet délétère des acides gras saturés et trans, effet } \\
\text { protecteur des acides gras mono-insaturés }\end{array}$ \\
\hline WHICAP (États-Unis) & 980 & $\geq 65$ & Lipides et acides gras & Maladie d'Alzheimer (MA) & $\begin{array}{l}\text { Effet délétère de l'énergie totale ingérée et des lipides } \\
\text { totaux seulement chez apoE } 4+ \\
\text { Pas d'association avec type d'acides gras }\end{array}$ \\
\hline
\end{tabular}

suggéré par deux études cas-témoin [22,23] et une étude en IRM [24]. II n'existe pas de données épidémiologiques longitudinales sur ce sujet. Une étude prospective récente a toutefois mis en évidence une diminution du risque de déclin cognitif chez les femmes consommant beaucoup de légumes verts à feuilles, riches en lutéine et zéaxantine, deux caroténoïdes xanthophylles [25].

Une seule étude d'intervention de supplémentation en vitamine $\mathrm{E}$ à $2000 \mathrm{U} /$ jour de patients atteints de MA s'est montrée positive [26] alors qu'un récent essai randomisé à la même dose chez des patients atteints de $\mathrm{MCl}$ s'est avéré négatif [27]. Une autre étude avec une association d'antioxydants n'a montré aucun effet sur le déclin cognitif de volontaires sains [28]. L'article de Chandra [29] qui rapportait des résultats positifs a été officiellement rétracté par le journal Nutrition en 2005 en raison d'incohérences graves dans les données présentées [30]. Une étude expérimentale menée chez des sujets atteints de MA tend à confirmer l'intérêt de l'association des vitamines $E$ et $C$ observée dans les études épidémiologiques pour lutter contre la peroxydation lipidique chez ces patients [31].

Les études épidémiologiques fournissent donc des résultats discordants quant à l'effet des anti-oxydants sur le vieillissement cérébral. Peu de données biologiques sont disponibles et certains nutriments, comme les caroténoïdes, ont été insuffisamment examinés. Apports alimentaires et statut biologique des individus ne sont pas analysés conjointement, d'où des difficultés dans l'interprétation des résultats. La plupart des études ont comparé des déments à des sujets normaux, sans apporter d'éléments nutritionnels ni biologiques sur le statut des sujets au stade $\mathrm{MCl}$ selon qu'ils évoluent ensuite ou non vers la démence. Des inconnues persistent quant aux doses efficaces, à l'intérêt des suppléments, et à l'interaction avec le tabagisme ou les caractéristiques génétiques. L'effet apparent des suppléments dans les étu- des d'observation pourrait être dû à des facteurs de confusion non contrôlés, notamment socioculturels. Le manque de prise en compte simultanée des autres nutriments, en particulier la consommation d'acides gras à longue chaîne très sensibles à la peroxydation, peut également expliquer certaines discordances entre études.

\section{Acides gras et vieillissement cérébral}

Les AGPI qui composent en grande partie les membranes des neurones sont indispensables au développement cérébral. À partir des acides gras essentiels que sont l'acide alphalinolénique $\mathrm{C} 18: 3(n-3)$ et l'acide linoléique $\mathrm{C} 18$ : 2(n-6), vont être synthétisés des dérivés à longue chaîne sous l'effet des élongases et des désaturases, en particulier l'acide arachidonique C20 : 4(n-6), l'EPA C20 : 5(n-3) et le DHA C22 : 6(n-3) qui est un constituant majeur de la membrane des neurones. Cette activité enzymatique diminue avec l'âge [32]. Le statut en DHA est alors beaucoup plus dépendant des apports nutritionnels [33].

Outre leur rôle dans la composition et la fluidité des membranes des neurones [34], plusieurs mécanismes pourraient expliquer les effets des AGPI au niveau cérébral. Les AGPI $\omega 3$ et $\omega 6$ ont des effets opposés sur le plan cardiovasculaire. Les AGPI 13 diminuent le risque d'arythmie cardiaque, ils ont aussi des effets antithrombotiques et anti-athérosclérose [35]. À côté de ces hypothèses vasculaires, le rôle des AGPI dans l'inflammation pourrait également expliquer leur effet sur le vieillissement cérébral. En effet, de nombreux travaux ont montré une association entre neuro-inflammation et pathologie neurodégénérative [36]. Les AGPI $\omega 3$ issus de l'alimentation pourraient prévenir la neurodégénérescence en régulant négativement la surexpression des cytokines inflammatoires qui apparaît au cours du vieillissement.
Enfin, les acides gras activent des récepteurs nucléaires spécifiques dénommés peroxisome proliferator-actived receptors (PPAR) et induisent ainsi la transcription de gènes codant pour des protéines et des enzymes impliquées dans la $\beta$-oxydation mitochondriale et peroxisomale [37]. Ces récepteurs forment des dimères avec le récepteur de l'acide rétinoïque (RXR). La capacité du DHA à se lier au RXR a été mise en évidence [38].

Peu d'études épidémiologiques longitudinales ont analysé les relations entre consommation de lipides et risque de démence((tableau 2). Les poissons gras sont très riches en AGPI $\omega 3$ à longue chaîne, notamment en DHA. L'effet protecteur de la consommation de poisson sur le risque de démence a été trouvé pour la première fois dans la Rotterdam Study [39], mais avec un suivi moyen de seulement 2,1 ans, ce qui fait que les participants avaient déjà pu modifier leur comportement alimentaire en raison de troubles cognitifs débutants. De plus, cette étude n'avait pas retrouvé de relation entre la consommation des différents types de lipides et le risque de démence [40]. Dans PAQUID, nous avons mis en évidence une corrélation inverse entre la fréquence de la consommation de poisson et le risque de développer une démence dans les 7 ans qui suivent [41]. Ce résultat a été reproduit dans l'étude CHAP [42], qui trouve également une relation avec la consommation totale d'AGPI $\omega 3$. Cette même étude a aussi montré un effet protecteur de la consommation de poisson contre le déclin cognitif en l'absence même de démence, mais $n^{\prime}$ a pas pu mettre en évidence d'effet spécifique des ACPI 103 à longue chaîne sur cette évolution [43]. Cependant, le questionnaire nutritionnel utilisé dans l'étude CHAP manque de fiabilité pour apprécier finement les apports en AGPI. L'association entre consommation d'AGPI et déclin cognitif était largement expliquée par la consommation concomitante de vitamine $E$ dans une autre analyse de cette cohorte, alors qu'au contraire un effet 
protecteur des acides gras mono-insaturés et un effet délétère des acides gras saturés et trans y étaient observés [44]. L'étude WHICAP n'a pas retrouvé de relation entre consommation d'AGPI et risque de MA, mais elle a mis en évidence un effet délétère de l'apport énergétique total et de la quantité de lipides ingérés chez les sujets porteurs de l'apoE4 [45]. Cette dernière étude soulève le problème des interactions complexes entre l'apport nutritionnel et les caractéristiques génétiques, en particulier pour les gènes impliqués dans le métabolisme des lipides et leur transport.

La Doetinchem Cohort Study a trouvé une association entre de meilleures performances cognitives, chez des adultes de 45 à 70 ans, et la consommation de poisson ou d'AGPI $\omega 3$ à longue chaîne (EPA et DHA) [46]. II s'agit d'une étude transversale, mais les participants n'avaient pas modifié leur comportement alimentaire au cours des 6 années précédentes. Dans l'étude $3 \mathrm{C}$ nous avons mis en évidence que les consommateurs réguliers de poisson avaient de meilleures performances cognitives globales, une meilleure santé perçue et un plus bas niveau de symptomatologie dépressive [47]. Ces analyses transversales concordent avec les études longitudinales tout en venant étayer I'hypothèse d'un effet des AGPI dans le bien-être via l'inflammation [48].

Les données biologiques vont dans le même sens que les résultats obtenus avec les données nutritionnelles. Dans EVA, nous avons observé une augmentation significative du risque de déclin cognitif avec le taux d'acides gras $\omega 6$ dans les membranes des globules rouges, et à l'inverse le taux d'acides gras 103 apparaissait potentiellement protecteur [49].

Aucune étude d'intervention évaluant l'effet des AGPI 103 dans la démence chez l'être humain n'a été publiée, à l'exception d'un petit essai qui a mis en évidence un effet positif $d^{\prime}$ une supplémentation en AGPI et vitamine $E$ sur la qualité de vie de la personne démente rapportée par l'aidant [50]. Un récent essai ayant montré la diminution des plaques séniles chez un modèle transgénique de souris Alzheimer âgée soumise à un régime enrichi en $\mathrm{DHA}$ suscite de grands espoirs [51].

\section{Conclusion}

Cette revue de la littérature montre que de nombreuses inconnues subsistent quant à la possibilité d'une prévention du vieillissement cérébral pathologique par la nutrition. Antioxydants et AGPI 103 à longue chaîne agissent en synergie. Leur effet conjoint doit donc être examiné simultanément, dans des études épidémiologiques longitudinales conduites dans de grands échantillons représentatifs de la population générale, comportant un suivi suf- fisamment long pour assurer l'antériorité du comportement alimentaire observé par rapport à l'apparition des troubles cognitifs. L'étude des interactions avec les caractéristiques génétiques, notamment l'apoE4, est balbutiante, alors qu'elle permettrait à la fois d'éclairer les mécanismes d'action de ces nutriments et de mieux cibler les bénéficiaires potentiels d'interventions. Devant les discordances des résultats d'études épidémiologiques et avant d'envisager des interventions nutritionnelles chez l'être humain, il importe de mieux étayer le mécanisme d'action des antioxydants et AGPI dans les différents stades du vieillissement cérébral. C'est l'objectif du programme de recherche COGINUT-3C (Cognition, anti-Oxydants, acides Gras, Inflammation et NUTrition: approche interdisciplinaire autour de l'étude 3 C) qui réunit des équipes de recherche épidémiologique et fondamentale de Bordeaux et Montpellier. Ses résultats pourront servir de base à de futures études d'intervention visant à évaluer l'impact de suppléments ou de recommandations nutritionnelles sur le risque de déclin cognitif et de démence.

\section{RÉFÉRENCES}

1. RAMAROSON H, HELMER C, BARBERGERGATEAU P, LETENNEUR L, DARTIGUES JF. Prévalence de la démence et de la maladie d'Alzheimer chez les personnes de 75 ans et plus: données réactualisées de la cohorte PAQUID. Rev Neurol 2003 ; 159 : 405-11.

2. WALKER LC, IBEGBU CC, TODD CW, et al. Emerging prospects for the disease-modifying treatment of Alzheimer's disease. Biochem Pharmacol 2005 ; 69 : 1001-8.

3. PETERSEN RC. Mild cognitive impairment as a diagnostic entity. I Intern Med 2004; 256 : 183-94.

4. LUCHSINGER JA, MAYEUX R. Dietary factors and Alzheimer's disease. Lancet Neurol 2004 . $3: 579-87$.

5. FLOYD RA, HENSLEY K. Oxidative stress in brain aging. Implications for therapeutics of neurodegenerative diseases. Neurobiol Aging $2002 ; 23$ : 795-807.

6. YOSHIDA S, SATO A, OKUYAMA H. Pathophysiological effects of dietary essential fatty acid balance on neural systems. Jpn / Pharmacol $1998 ; 77: 11-22$.

7. COMMENGES D, SCOTET V, RENAUD S, JACQMIN-GADDA H, BARBERGER-GATEAU P, DARTIGUES J-F. Intake of flavonoids and risk of dementia. Eur / Epidemiol 2000 ; 16 : 357-63.

8. BERR C, BALANSARD B, ARNAUD J, ROUSSEL AM, ALPEROVITCH A. Cognitive decline is associated with systemic oxidative stress : the EVA study. Etude du Vieillissement Arteriel. I Am Geriatr Soc 2000 ; 48 : 1285-91.
9. THREE-CITY STUDY GROUP. Vascular factors and risk of dementia : design of the Three-City Study and baseline characteristics of the study population. Neuroepidemiology 2003; 22: 316-25.

10. DESCHAMPS V, ASTIER $X$, BARBERGERGATEAU P, FERRY M. Etat nutritionnel et état de santé des personnes âgées. Etude comparative de deux enquêtes longitudinales françaises: PAQUINUT en Dordogne et EURONUT/ SENECA à Romans. Med Nutr $2001 ; 37$ : 259 66.

11. COYLE JT, PUTTFARCKEN P. Oxidative stress, glutamate, and neurodegenerative disorders. Science 1993 ; 262 : 689-95.

12. MONTINE TJ, NEELY MD, QUINN JF, et al. Lipid peroxidation in aging brain and Alzheimer's disease. Free Radic Biol Med 2002; 33 : 620-6.

13. MARKESBERY WR, KRYSCIO RI, LOVELL MA, MORROW JD. Lipid peroxidation is an early event in the brain in amnestic mild cognitive impairment. Ann Neurol 2005 ; 58 : 730-5.

14. ENGELHART MJ, GEERLINGS MI, RUITENBERG $A$, et al. Dietary intake of antioxidants and risk of Alzheimer disease. JAMA 2002; 287: 3223-9.

15. MORRIS MC, EVANS DA, BIENIAS IL, TANGNEY CC, WILSON RS. Vitamin E and cognitive decline in older persons. Arch Neurol 2002; 59 : 1125-32.

16. LUCHSINGER JA, TANG MX, SHEA S, MAYEUX R. Antioxidant vitamin intake and risk of Alzheimer disease. Arch Neurol 2003; 60 : 203-8.

17. MORRIS MC, BECKETT LA, SCHERR PA, et al. Vitamin $E$ and vitamin $C$ supplement use and risk of incident Alzheimer disease. Alz Dis Assoc Disorder 1998 ; 12 : 121-6.

18. ZANDI PP, ANTHONY IC, KHACHATURIAN AS, et al. Reduced risk of Alzheimer disease in users of antioxidant vitamin supplements - The Cache County Study. Arch Neurol $2004 ; 61$ : 82-8.

19. GRODStEIN F, CHEN J, WILLETT WC. Highdose antioxidant supplements and cognitive function in community-dwelling elderly women. Am J Clin Nutr 2003 ; 77 : 975-84.

20. LAURIN D, FOLEY D], MASAKI KH, WHITE LR, LAUNER LJ. Vitamin E and $C$ supplements and risk of dementia. JAMA $2002 ; 288$ : 2266-8.

21. HELMER C, PEUCHANT E, LETENNEUR L, et al. Association between antioxidant nutritional indicators and the incidence of dementia: results from the PAQUID prospective cohort study. Eur / Clin Nutr $2003 ; 57: 1555-61$.

22. RINALDI P, POLIDORI MC, METASTASIO A, et al. Plasma antioxidants are similarly depleted in mild cognitive impairment and in Alzheimer's disease. Neurobiol Aging 2003; 24 : 915-9. 
23. POLIDORI MC, MATTIOLI P, ALDRED $S$, et al. Plasma antioxidant status, immunoglobulin $\mathrm{g}$ oxidation and lipid peroxidation in demented patients: relevance to Alzheimer disease and vascular dementia. Dement Geriatr Cogn Disord $2004 ; 18: 265-70$.

24. DEN HEIJER T, LAUNER LJ, DE GROOT JC, et al. Serum carotenoids and cerebral white matter lesions : the Rotterdam scan study. I Am Geriatr Soc $2001 ; 49$ : 642-6.

25. KANG JH, ASCHERIO A, GRODSTEIN F. Fruit and vegetable consumption and cognitive decline in aging women. Ann Neurol 2005; $57: 713-20$.

26. SANO M, ERNESTO C, THOMAS RG, et al. A controlled trial of selegiline, alpha-tocopherol, or both as treatment for Alzheimer's disease. The Alzheimer's Disease Cooperative Study. N Engl J Med 1997 ; 336 : 1216-22.

27. PETERSEN RC, THOMAS RG, GRUNDMAN M, et al. Vitamin $\mathrm{E}$ and donepezil for the treatment of mild cognitive impairment. $N$ Engl / Med $2005 ; 352$ : 2379-88.

28. SMITH A, CLARK R, NUTT D, HALLER J HAYWARD S, PERRY K. Anti-oxidant vitamins and mental performance of the elderly. Hum Psychopharmacol Clin Exp 1999 ; 14 : 459-71.

29. CHANDRA RK. Effect of vitamin and traceelement supplementation on cognitive function in elderly subjects. Nutrition $2001 ; 17$ : 709-12.

30. MEGUID MM. Retraction. Nutrition 2005 ; 21 : 286.

31. KONTUSH A, MANN U, ARLT S, et al. Influence of vitamin $E$ and $C$ supplementation on lipoprotein oxidation in patients with Alzheimer's disease. Free Radic Biol Med 2001 ; 31 : 345-54.

32. BURDGE GC, FINNEGAN YE, MINIHANE AM, WILLIAMS CM, WOOTTON SA. Effect of altered dietary n-3 fatty acid intake upon plasma lipid fatty acid composition, conversion of [13C]alpha-linolenic acid to longer-chain fatty acids and partitioning towards beta-oxidation in older men. Br / Nutr $2003 ; 90: 311-21$.
33. MUSKIET FA, FOKKEMA MR, SCHAAFSMA A, BOERSMA ER, CRAWFORD MA. Is docosahexaenoic acid (DHA) essential? Lessons from DHA status regulation, our ancient diet, epidemiology and randomized controlled trials. I Nutr $2004 ; 134$ : 183-6.

34. YEHUDA S, RABINOVITZ S, CARASSO RL, MOSTOFSKY DI. The role of polyunsaturated fatty acids in restoring the aging neuronal membrane. Neurobiol Aging 2002 ; 23 : 84353.

35. KRIS-ETHERTON PM, HARRIS WS, APPEL LJ. For the nutrition committee. Fish consumption, fish oil, omega- 3 fatty acids, and cardiovascular disease. Circulation $2002 ; 106$ : 2747-57.

36. FLOYD RA. Neuroinflammatory processes are important in neurodegenerative diseases: an hypothesis to explain the increased formation of reactive oxygen and nitrogen species as major factors involved in neurodegenerative disease development. Free Radic Biol Med $1999 ; 26$ : 1346-55.

37. ALESSANDRI JM, GUESNET P, VANCASSEL $S$, et al. Polyunsaturated fatty acids in the central nervous system: evolution of concepts and nutritional implications throughout life. Reprod Nutr Dev 2004 ; 44 : 509-38

38. DE URQUIZAAM, LIU S, SJOBERG M, et al. Docosahexaenoic acid, a ligand for the retinoid $X$ receptor in mouse brain. Science $2000 ; 290$ : 2140-4.

39. KALMIJN S, LAUNER LJ, OTT A, WITTEMAN JCM, HOFMAN A, BRETELER MMB. Dietary fat intake and the risk of incident dementia in the Rotterdam study. Ann Neurol 1997 ; 42 : 776 82.

40. ENGELHART M), GEERLINGS MI, RUITENBERC $A$, et al. Diet and risk of dementia : Does fat matter? The Rotterdam Study. Neurology $2002 ; 59$ : 1915-21.

41. BARBERGER-GATEAU P, LETENNEUR L, DESCHAMPS V, PERES K, DARTIGUES J-F, RENAUD $S$. Fish, meat, and risk of dementia : cohort study. Br Med / 2002 ; 325 : 932-3.
42. MORRIS MC, EVANS DA, BIENIAS IL, et al. Consumption of fish and n-3 fatty acids and risk of incident Alzheimer disease. Arch Neurol $2003 ; 60$ : 940-6.

43. MORRIS MC, EVANS DA, TANGNEY CC, BIENIAS JL, WILSON RS. Fish consumption and cognitive decline with age in a large community study. Arch Neurol 2005; (62.12.noc50161).

44. MORRIS MC, EVANS DA, BIENIAS JL, TANGNEY CC, WILSON RS. Dietary fat intake and 6-year cognitive change in an older biracial community population. Neurology $2004 ; 62$ : 1573-9.

45. LUCHSINGER JA, TANG MX, SHEA S, MAYEUX R. Caloric intake and the risk of Alzheimer disease. Arch Neurol 2002 ; 59 : 1258-63.

46. KALMIJN S, VAN BOXTEL MP, OCKE M, VERSCHUREN WM, KROMHOUT D, LAUNER LJ. Dietary intake of fatty acids and fish in relation to cognitive performance at middle age. Neurology $2004 ; 62: 275-80$.

47. BARBERGER GATEAU P, JUTAND MA, LETENNEUR L, LARRIEU S, TAVERNIER B, BERR C. Correlates of regular fish consumption in French elderly community dwellers : data from the Three-City study. Eur / Clin Nutr 2005 ; 59 : 817-25.

48. CAPURON L, DANTZER R. Cytokines and depression: the need for a new paradigm. Brain Behav Immun 2003 ; 17(Suppl 1) : S119. S124.

49. HEUDE B, DUCIMETIERE P, BERR C. Cognitive decline and fatty acid composition of erythrocyte membranes - The EVA Study. Am / Clin Nutr $2003 ; 77: 803-8$.

50. YEHUDA S, RABINOVTZ S, CARASSO R, MOSTOFSKY DI. Essential fatty acids preparation (SR-3) improves Alzheimer's patients quality of life. Int J Neurosci 1996 ; 87 : 141-9.

51. LIM GP, CALON F, MORIHARA T, et al. A diet enriched with the Omega-3 fatty acid docosahexaenoic acid reduces amyloid burden in an aged Alzheimer mouse model. I Neurosci $2005 ; 25: 3032-40$. 